\title{
Moving towards genomic therapy for amyotrophic lateral sclerosis
}

Superoxide dismutase 1 (SOD1) gene mutations account for up to $20 \%$ of cases of familial amyotrophic lateral sclerosis (ALS), and this gene could represent an important therapeutic target. Two new reports in The New England Journal of Medicine provide early indications that knockdown of SOD 1 expression is feasible and warrants further exploration in people with SOD1 ALS.

The first study used the antisense oligonucleotide (ASO) tofersen to target SOD1. "ASOs bind to the target mRNA and activate RNAse $\mathrm{H}$, which degrades the mRNA," explains lead author Timothy Miller. "As mutations in SOD1 cause a toxic gain of function, lowering the levels of SOD1 protein is predicted to be therapeutic."

The phase I/II trial included 50 patients with SOD1 ALS, 48 of whom completed the treatment course. The participants were randomly assigned to tofersen $(20,40,60$ or $100 \mathrm{mg})$ or placebo, administered intrathecally in five doses over a 12 -week period.

In the patients who received the highest dose of tofersen, SOD1 levels were substantially reduced in the cerebrospinal fluid (CSF). Although the trial was not sufficiently powered to demonstrate clinical efficacy, some of the treated patients also showed evidence of improvements in clinical function and muscle strength.

"We are currently studying the efficacy and safety of tofersen in a phase III study," says Miller. "The study is enrolling a mix of fast-progressing and slow-progressing patients so we can fully understand the potential of the drug."

In the second study, two patients with SOD1 ALS received a SOD1targeting microRNA, delivered into

the CSF via an adeno-associated virus (AAV) vector. "A potential advantage of AAV-microRNA over ASOs is the duration of action," explains corresponding author Robert Brown. "ASOs have to be administered a few times a year, whereas in principle a single AAV-microRNA treatment should persist for years."

Like the other trial, this study was not designed to demonstrate efficacy. However, one of the patients showed apparent preservation of strength in one leg. Post-mortem spinal cord tissue from this patient contained lower-than-average SOD1 levels. In both patients, the treatment provoked an immune response, which could be dampened with immunosuppressive drugs.

"We are now planning a larger trial in $~ 30$ patients," says Brown. "We are also likely to incorporate a version of our immunosuppression protocol into other AAV trials."

Heather Wood

reduced in the cerebrospinal fluid

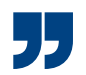

\section{Aspirin and ibuprofen could lower risk of $L R R K 2$ Parkinson disease}

Regular use of non-steroidal antiinflammatory drugs (NSAIDs) might reduce the penetrance of $L R R K 2$ mutations associated with Parkinson disease (PD), according to new research. The findings raise the possibility of a simple diseasemodifying treatment for people with $L R R K 2$ variants.

Inflammation is thought to be involved in the pathogenesis of PD, and use of NSAIDs, such as aspirin and ibuprofen, has previously been associated with a lower risk of PD. In the new study, the researchers investigated this association specifically among people with LRRK2 mutations.

Autosomal dominant mutations in LRRK2 are the most common cause of Mendelian PD, though the penetrance of these mutations is low at $\sim 30 \%$ at age 80 years. Other variants in LRRK2 are also associated with an increased risk of PD. LRRK2 encodes leucine-rich repeat kinase 2 , which is expressed in immune cells and influences inflammatory pathways. This role in inflammation provided the basis for investigating the effects of NSAIDs in people with LRRK2 mutations.

The study involved members of two international cohorts: the Parkinson's Disease Genetic and Environmental Modifiers cohort, and the Michael J. Fox Foundation LRRK2 Cohort Consortium. In total, 577 individuals with pathogenic or risk variants in LRRK2 were included -259 had PD and 318 were asymptomatic. Participants were asked whether they had ever taken ibuprofen-based medication, aspirin or other anti-inflammatory medications regularly, defined as two or more pills weekly for $>6$ months.
Regular use of NSAIDs was more common among asymptomatic carriers of $L R R K 2$ variants than among symptomatic carriers. Regression analysis indicated that regular NSAID use is associated with a lower risk of PD among people with LRRK2 mutations. The association was true for risk variants as well as pathogenic variants.

"Our results support the hypothesis that aspirin and ibuprofen can reduce the risk of PD manifestation among LRRK2 carriers," says lead author Marta San Luciano. "Anti-inflammatory drugs may be useful as disease-modifying treatments in LRRK2-PD, and the ability to identify an at-risk population makes interventions in this subgroup particularly feasible. However, these findings need to be replicated in other cohorts and in longitudinal studies, which is our planned next step."

lan Fyfe

ORIGINAL ARTICLE San Luciano, M. et al. Nonsteroidal anti-inflammatory use and LRRK2 Parkinson's disease penetrance. Mov. Disord. https://doi.org/10.1002/mds.28189 (2020) RELATED ARTICLE Tolosa, E. et al. LRRK2 in Parkinson disease: challenges of clinical trials. Nat. Rev. Neurol. 16, 97-107 (2020) 